\title{
A Simplified Skeletal Maturity Scale and Thumb Ossification Composite Index to Assess Skeletal Maturity and Predict Height Velocity in Japanese Females with Adolescent Idiopathic Scoliosis
}

\author{
Masaaki Chazono ${ }^{1 / 2)}$ and Shintaro Obata ${ }^{122}$ \\ 1) Department of Orthopaedic Surgery, NHO Utsunomiya National Hospital, Tochigi, Japan \\ 2) Department of Orthopaedic Surgery, The Jikei University School of Medicine, Tokyo, Japan
}

\begin{abstract}
:
Introduction: Since Little et al. first reported that peak height velocity (PHV) could be described as a reliable clinical marker for the prediction of remaining growth and curve progression of adolescent idiopathic scoliosis (AIS) in clinical practice, much attention has been paid to PHV as a possible predictor of curve progression in patients with AIS. However, PHV itself is only identified retrospectively, so its value is not available at the first outpatient clinic visit. Using the simplified skeletal maturity scale (SSMS) and the thumb ossification composite index (TOCI) staging systems, this study aims to assess skeletal maturity and predict height velocity (HV) in Japanese female patients with AIS.

Methods: This study involved 95 female patients with AIS, ranging from 9 to 17 years old. A standing AP radiograph of the entire spine and a hand radiograph were retrospectively obtained to establish the skeletal maturity stage in accordance with the SSMS and TOCI systems. Height measurements were recorded at each visit; HV was calculated as the height change $(\mathrm{cm})$ divided by the time interval (years).

Results: The TOCI stage rating increased identically to the SSMS stage rating increase. The chi-square test showed that there was a significant correlation between the two scoring systems $\left(\chi^{2}=720.4\right)$. The Cramer V correlation also demonstrated a very strong correlation (Cramer $\mathrm{V}=0.62$ ). Regarding the relationship between HV and each SSMS and TOCI stage, HV decreased as SSMS and TOCI stages increased. $\eta^{2}$ equaled to 0.67 in both groups and displayed a strong correlation between HV and SSMS and TOCI stages.

Conclusions: These findings suggest that evaluation of the rate of HV using these radiological assessments is of supreme importance for determining the status of pubertal maturity and predicting the remaining amount of adolescent growth at the outpatient clinic visit.
\end{abstract}

Keywords:

Adolescent idiopathic scoliosis, Peak height velocity, Simplified skeletal maturity scale, Thumb ossification composite index

Spine Surg Relat Res 2021; 5(4): 244-251 dx.doi.org/10.22603/ssrr.2020-0176

\section{Introduction}

Generally, adolescent idiopathic scoliosis (AIS) progression is correlated with skeletal growth; it peaks during the growth period and then slows and stabilizes, in most cases, toward the end of skeletal maturity ${ }^{1}$. The close connection between AIS curve progression and skeletal growth provides a strong impetus to find a simple and reliable approach to assess skeletal maturity. In 2000, Little et al. first reported that most curves progressed with the timing of peak height velocity (PHV) ${ }^{1}$. They found that 60 of 88 patients had a scoliosis curve greater than $30^{\circ}$ at $\mathrm{PHV}$, and in 50 of 60 patients, the curve had progressed to $45^{\circ}$ or greater. Around a decade ago, Sanders's simplified skeletal maturity scale (SSMS) using a hand X-ray was developed and derived from the original Tanner-Whitehouse-3 radius, ulna, and short bones of the hand score ${ }^{2}$. They reported that if a female patient had capped distal phalangeal epiphyses, she probably had reached PHV and if her distal phalangeal epiphyses were closed, she had likely already achieved PHV.

Corresponding author: Masaaki Chazono, chazono@pop02.odn.ne.jp

Received: September 25, 2020, Accepted: November 30, 2020, Advance Publication: January 12, 2021

Copyright (C) 2021 The Japanese Society for Spine Surgery and Related Research 
Additionally, compared with Risser staging, the SSMS is simpler and more reproducible, has a substantial correlation with the curve acceleration phase, and is a good predictor of $\mathrm{PHV}^{3)}$. Therefore, the timing of the maximum adolescent height velocity (HV) is the most essential skeletal maturity indicator in patients with scoliosis ${ }^{4)}$. In recent years, Hung et al. developed a new thumb classification on the basis of the epiphyses of three bones: the distal and proximal phalanges together with the adductor sesamoid bone. This is referred to as the thumb ossification composite index (TOCI) ${ }^{5}$. Its abbreviated form is pronounced "tosi" rather than "tochi." The TOCI classification is intended to further simplify the SSMS system by focusing on the morphology of a minimum number of essential hand ossifications. Using the SSMS and TOCI staging systems, this study aims to assess skeletal maturity and prospectively predict the value of $\mathrm{HV}$ in a cohort of Japanese female patients.

\section{Materials and Methods}

\section{Patient population}

This retrospective cohort study was approved by the Institutional Review Board of the authors' affiliated institutions. In this study, 95 female patients with AIS were included, who were skeletally immature and had been followed-up at our hospital during their peripubertal growth period until skeletal maturity was reached. Outpatient clinic visits occurred at least four times and were, on average, 4 months apart. The mean age of the patients at the time of their first visit was 13.5 years, and their overall range in age was 9 to 17 years. Their mean Cobb angle at the initial visit was $23.6^{\circ}\left(10^{\circ}-45^{\circ}\right)$. There were 70 patients in the observation group, 22 in the brace treatment group, and three in the surgery group at the final follow-up visit. There were 64 patients with curve-type Lenke 1, 10 with Lenke 2, three with Lenke 3, 16 with Lenke 5, and two with Lenke 6, and most patients had a single thoracic curve (Table 1).

\section{SSMS stages and TOCI stages}

The SSMS system is stratified into eight stages ${ }^{6}$. The juvenile slow stage (SSMS 1) is the stage before the beginning of the adolescent growth spurt. In radiographs, digital epiphyses are not covered. The preadolescent slow stage (SSMS 2) is the stage during which the adolescent growth spurt has started but before PHV has occurred. All digital epiphyses are covered. The adolescent early rapid stage (SSMS 3) is the stage in which the scoliosis curves start their acceleration phase, corresponding with the timing of PHV. Most digits are capped. The second through fifth metacarpal epiphyses are wider than their metaphyses. In the adolescent late rapid stage (SSMS 4), scoliosis continues to increase rapidly. Any of the distal phalangeal physes are beginning to close. The adolescent early steady stage (SSMS 5 ) is the stage where girls are still typically in Risser stage 0 , but at this stage, the triradiate cartilage is closed. All dis-
Table 1. Patients Demographic Data.

\begin{tabular}{llr}
\hline Mean age at first visit (years) & 13.5 \\
\hline Mean Cobb angle at first visit $\left(^{\circ}\right)$ & 23.6 \\
\hline \multirow{3}{*}{ Treatment outcome } & Observation & 70 \\
& Brace & 22 \\
& Surgery & 3 \\
\hline \multirow{4}{*}{ Lenke classification } & Lenke 1 & 64 \\
& Lenke 2 & 10 \\
& Lenke 3 & 3 \\
& Lenke 5 & 16 \\
& Lenke 6 & 2 \\
\hline
\end{tabular}

tal phalangeal physes are closed, whereas middle and proximal phalangeal physes are open. During the adolescent late steady stage (SSMS 6), a Risser sign is usually positive. Menarche usually has occurred. Middle or proximal phalangeal physes are closing. The early mature stage (SSMS 7) is the stage that usually corresponds to Risser stage 4, and scoliosis can still occur. Radiological findings demonstrate that only the distal radial physis is open. Metacarpal physes scars may be present. Finally, the mature stage (SSMS 8) is the stage that corresponds to Risser stage 5 and the completion of growth in most cases. The distal radial physis is completely closed (Table $2 \mathrm{a}$ ).

The TOCI system is also divided into eight stages ${ }^{5)}$. Fig. 1 shows the representative images of each TOCI stage in our series. The juvenile stage (TOCI 1) is the stage before the beginning of the adolescent growth spurt. The thumb's proximal phalangeal epiphysis is not wider than the metaphysis, and the adductor sesamoid is absent on radiographs. The prepubertal stage (TOCI 2) is still a stage before the adolescent growth spurt. The thumb's proximal phalangeal epiphysis is wider than the metaphysis but without capping. The pubertal onset stage (TOCI 3) indicates the timing of pubertal onset. A roundish covered ulnar corner of the thumb's proximal phalangeal epiphysis is observed, and an ossified adductor sesamoid bone appears. The ascending phase (TOCI 4) is close to PHV. Early capping of the ulnar corner of the thumb's proximal phalangeal epiphysis is observed. TOCI 5 is coincident with a critical PHV period. Advanced capping of the ulnar corner of the thumb's proximal phalangeal epiphysis is observed. The early descending stage (TOCI 6) is the stage when menarche commonly starts. The thumb's distal phalangeal epiphysis is completely fused. The middle descending stage (TOCI 7) shows further slowing of the growth rate. The thumb's proximal phalangeal epiphysis is partially fused. Lastly, the late descending stage (TOCI 8) confirms late maturity. The thumb's proximal phalangeal epiphysis is completely fused (Table 2 b). These skeletal maturity systems cover from the juvenile stage to the mature status in the adolescent growth period. According to their study, SSMS 7 or above and TOCI 8 are regaraded to be the mature status: mean absolute remaining growth $(\mathrm{cm})$, mean remaining growth period (years), and 
Table 2.

a. SSMS Stage during Bone Growth Phases

\begin{tabular}{cll}
\hline SSMS & \multicolumn{1}{c}{ Stage } & \multicolumn{1}{c}{ Radiographic features } \\
\hline 1 & Juvenile slow & Digital epiphyses are not covered \\
2 & Preadolescent slow & All digital epiphyses are covered \\
3 & Adolescent rapid (Early) & Most digits are capped. 2nd-5th metacarpal epiphyses are wider than their metaphyses \\
4 & Adolescent rapid (Late) & Any of distal phalangeal physes are beginning to close \\
5 & Adolescent steady (Early) & All distal phalangeal physes are closed, while others are open \\
6 & Adolescent steady (Late) & Middle or proximal phalangeal physes are closing \\
7 & Early mature & Only distal radial physes is open. Metacarpal physeal scars may be present \\
8 & Mature & Distal radial physis is completely closed \\
\hline
\end{tabular}

SSMS Simplified Skeletal Maturity Scale

b. TOCI Stage during Bone Growth Phases

\begin{tabular}{|c|c|c|}
\hline TOCI & Stage & Radiographic Features \\
\hline 1 & Juvenile & Thumb's proximal phalangeal epiphysis is not wider than the metaphysis. Adductor sesamoid bone is absent \\
\hline 2 & Prepuberty & Thumb's proximal phalangeal epiphysis is wider than the metaphysis but without capping \\
\hline 3 & Pubertal onset & $\begin{array}{l}\text { Roundish covered ulnar corner of thumb's proximal phalangeal epiphysis. An ossified adductor sesamoid } \\
\text { bone appeared }\end{array}$ \\
\hline 4 & Ascending & Early capping of ulnar corner of the thumb's proximal phalangeal epiphysis is observed \\
\hline 5 & PHV & Advanced capping of the ulnar corner of the thumb's proximal phalangeal epiphysis is seen \\
\hline 6 & Discending (Early) & Thumb's distal phalangeal epiphysis completely fused \\
\hline 7 & Discending (Middle) & Thumb's proximal phalangeal epiphysis partially fused \\
\hline 8 & Discending (Late) & Thumb's proximal phalangeal epiphysis completely fused \\
\hline
\end{tabular}

TOCI Thumb Ossification Composite Index, $P H V$ Peak Height Velocity
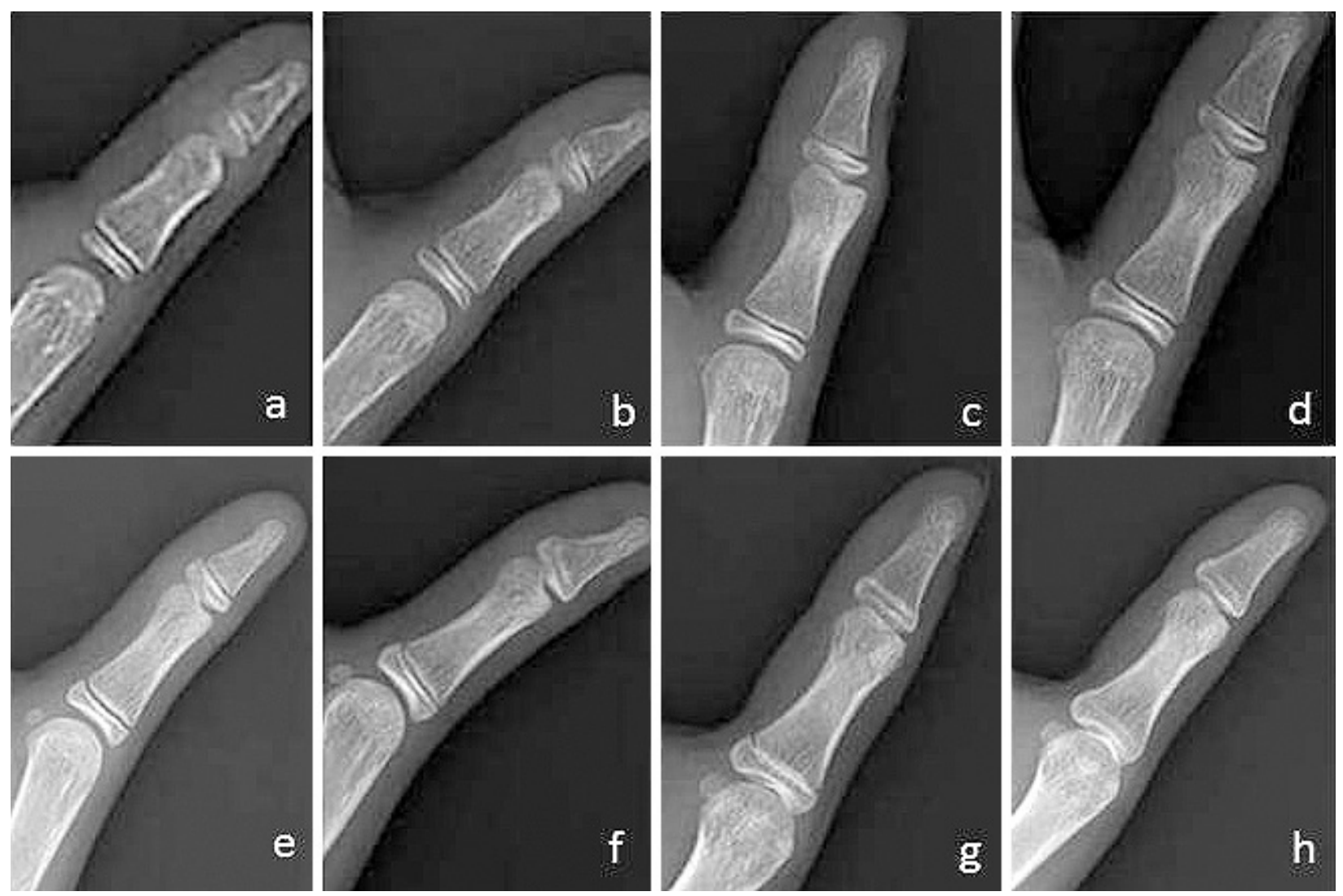

Figure 1. Representative images of TOCI. a: TOCI 1, b: TOCI 2, c: TOCI 3, d: TOCI 4, e: TOCI 5, f: TOCI 6, g: TOCI 7, h: TOCI 8. For details, please see Table 2b. TOCI=Thumb Ossification Composite Index

mean HV (cm/year) were respectively $2.7 \mathrm{~cm}, 1.7$ years, and $1.9 \mathrm{~cm} /$ year in SSMS 7 and $2.5 \mathrm{~cm}, 1.7$ years, and $1.7 \mathrm{~cm} /$ year in TOCI $8^{5}$. Therefore, the mature status is also defined as SSMS 7 or above and TOCI 8 in the present study. 
Table 3. SSMS-stage Rating That Matches Each TOCI-stage.

\begin{tabular}{rcccccrrrr}
\hline & TOCI 1 & TOCI 2 & TOCI 3 & TOCI 4 & TOCI 5 & TOCI 6 & TOCI 7 & TOCI 8 & Total \\
\hline SSMS 1 & 6 & & & & & & & & 6 \\
SSMS 2 & 4 & 3 & & & & & & & 7 \\
SSMS 3 & & 3 & 7 & 22 & 16 & 1 & & & 49 \\
SSMS 4 & & & & 1 & 11 & 13 & & 1 & 26 \\
SSMS 5 & & & & & 1 & 8 & 8 & 2 & 19 \\
SSMS 6 & & & & & & 3 & 10 & 5 & 18 \\
SSMS 7 & & & & & & & 7 & 76 & 83 \\
SSMS 8 & & & & & & & & 58 & 58 \\
\hline Total & 10 & 6 & 7 & 23 & 28 & 25 & 25 & 142 & 266 \\
\hline
\end{tabular}

SSMS Simplified Skeletal Maturity Scale, TOCI Thumb Ossification Composite Index

\section{Study protocol}

A hand radiograph and a standing AP whole spine radiograph were retrospectively obtained by one of the authors (M.C.) to establish skeletal maturity staging according to SSMS and TOCI systems. Height measurement was subsequently recorded. HV was calculated as the change in height (cm) divided by the time interval (years) between the 6 and 12 month visits, averaging at 8 months. The maximum value of $\mathrm{HV}$ was defined as the PHV in the present study. First, the number of the SSMS stage matching with each TOCI stage was tabulated to distribute their frequencies. Second, the mean, median, standard deviation, and $95 \%$ confidence interval of the SSMS stage corresponding to each TOCI stage were calculated to determine the relationship between the two sets of scores. Third, the correlation of SSMS and TOCI stages with HV was examined and calculated. Fourth, regarding the reliability of SSMS and TOCI classification, the principal examiner (M.C.) scored all radiographs and another examiner, who was not involved in the study, scored those radiographs, independently. The two examiners were blinded to the chronological age of the patients and performed the ratings. After 1 month, the two reviewers repeated the ratings using the same set of radiographs. The reliability of the SMMS and TOCI classifications were expressed by intraclass and interclass correlation coeffcients.

\section{Statistical analysis}

During statistical analysis, the chi-square test and the Cramer V correlation were utilized to identify the interchangeability between the categorical SSMS and TOCI stages. The correlation between the SSMS/TOCI stages and HV was calculated on the intraclass and interclass variance and expressed as the correlation ratio $\left(\eta^{2}\right)$, respectively. The strength of Cramer $\mathrm{V}$ and $\eta^{2}$ correlation indicate 0.5-1.0 as strongest, $0.25-0.5$ as moderate, $0.1-0.25$ as mild, and $<0.1$ as no correlation. These statistical analyses were performed using BellCuve for Excel 3.21 (Social Survey Research Information Co., Ltd., Tokyo, Japan). The significance level was $\mathrm{p}<0.05$.
Table 4. Distribution of the SSMS Score with Each TOCI-stage.

\begin{tabular}{llllc}
\hline & Mean & Median & \multicolumn{1}{c}{ SD } & 95\% CI \\
\hline TOCI 1 & 1.4 & 1 & 0.52 & $1.07-1.72$ \\
TOCI 2 & 2.5 & 2.5 & 0.55 & $2.06-2.94$ \\
TOCI 3 & 3 & 3 & 0 & 3 \\
TOCI 4 & 3.04 & 3 & 0.21 & $2.96-3.13$ \\
TOCI 5 & 3.46 & 3 & 0.58 & $3.25-3.68$ \\
TOCI 6 & 4.52 & 4 & 0.77 & $4.20-4.84$ \\
TOCI 7 & 5.96 & 6 & 0.79 & $5.65-6.27$ \\
TOCI 8 & 7.32 & 7 & 0.68 & $7.21-7.44$ \\
\hline
\end{tabular}

SSMS Simplified Skeletal Maturity Scale, TOCI Thumb Ossification Composite Index

\section{Results}

\section{SSMS stage ratings in comparison with TOCI stage}

A total of 6118 epiphyses obtained from 266 hand radiographs were scored for 95 consecutive patients. Table 3 summarizes the exact SSMS stage rating that matches each TOCI stage. SSMS stage ratings increased in parallel with increases in TOCI stage ratings. Both TOCI 4 and TOCI 5 overlapped by $78 \%$ (38/49) with SSMS 3, suggesting that SSMS 3 is consistent with TOCI 4 or TOCI 5. Similarly, TOCI 7 overlapped by $56 \%(10 / 18)$ with SSMS 6, and TOCI 8 overlapped by 92\% (76/83) with SSMS 7, suggesting that TOCI 8 corresponds with SSMS 7. Both SSMS stage ratings and TOCI stage ratings were strongly associated as confirmed via the chi-square test and Cramer V correlation test $\left(\chi^{2}=720.4\right.$ and Cramer $\left.V=0.62\right)$, with a statistical significance of $\mathrm{p}<0.01$.

\section{Distribution of the SSMS score with each TOCI stage}

Table 4 summarizes the mean, median, standard deviation, and $95 \%$ confidence interval calculated for each TOCI stage. TOCI 1 was consistent with SSMS 1. TOCI 3 to TOCI 5 matched SSMS 3. Subsequently, TOCI 6, TOCI 7, and TOCI 8 matched SSMS 4, SSMS 6, and SSMS 7, respectively. This result demonstrated that TOCI stage can predict SSMS stage. 


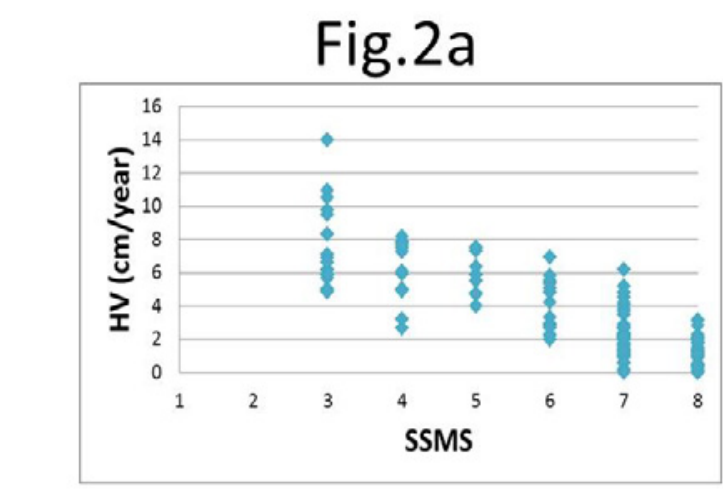

\begin{tabular}{|c|c|c|c|c|c|c|}
\hline & SSMS 3 & SSMS 4 & SSMS 5 & SSMS 6 & SSMS 7 & SSMS 8 \\
\hline Mean & 7.4 & 6.2 & 5.8 & 4.1 & 2.3 & 1.3 \\
\hline SD & 2.6 & 1.9 & 1.3 & 1.6 & 1.4 & 0.8 \\
\hline $\mathbf{n}$ & 18 & 12 & 8 & 13 & 40 & 25 \\
\hline
\end{tabular}

Fig. $2 b$

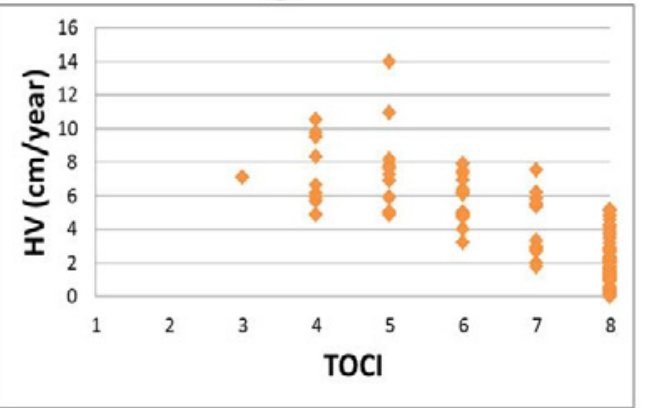

\begin{tabular}{|c|c|c|c|c|c|c|}
\hline & TOCI 3 & TOCI 4 & TOCI 5 & TOCI 6 & TOCI 7 & TOCI 8 \\
\hline Mean & 7.1 & 7.3 & 7.4 & 5.7 & 4.3 & 1.7 \\
\hline SD & & 2.0 & 2.7 & 1.4 & 1.9 & 1.3 \\
\hline n & 1 & 10 & 12 & 14 & 12 & 67 \\
\hline
\end{tabular}

Figure 2. Correlation of Height velocity (HV) with each SSMS (Fig. 2a) and TOCI (Fig. 2b) stage.

$\mathrm{HV}=$ height velocity, SSMS=Simplified Skeletal Maturity Scale, TOCI=Thumb Ossification Composite Index

\section{HV vs. each SSMS and TOCI stage}

SSMS 3, SSMS 4, SSMS 5, SSMS 6, SSMS 7, and SSMS 8 corresponded with mean HVs of 7.4, 6.2, 5.8, 4.1, 2.3 , and $1.3 \mathrm{~cm} /$ year, respectively (Fig. 2a). By contrast, TOCI 4, TOCI 5, TOCI 6, TOCI 7, and TOCI 8 corresponded with mean HVs of 7.3, 7.4, 5.7, 4.3 , and $1.7 \mathrm{~cm} /$ year, respectively (Fig. 2b), showing that the degree of mean HV decreased as SSMS and TOCI stages increased. These results showed that an $\mathrm{HV}$ of $7.4 \mathrm{~cm} /$ year was associated with both SSMS 3 and TOCI 5, indicating that both values were possibly the PHV. Concerning the statistical relationship, intraclass variance, interclass variance, and $\eta^{2}$ were 280.2, 576.5, and 0.67 for HV vs. SSMS stage, respectively, whereas those values were 282.3, 562.8, and 0.67 for HV vs. TOCI stage, respectively, displaying a strong correlation between HV and SSMS and TOCI stages. When these two line graphs were shown and superimposed based on the HV values, each SSMS stage was correlated with each TOCI stage as follows: SSMS 3 corresponded with TOCI 5, SSMS 4 and SSMS 5 with TOCI 6, SSMS 6 with TOCI 7, and finally, SSMS 7 with TOCI 8 (Fig. 3).

\section{Reliability test}

Two clinical professionals showed excellent intraclass and interclass agreement for the SSMS and TOCI classification (Table 5).

\section{Discussion}

For three decades, various skeletal and non-skeletal maturity indicators have been advocated as prognostic factors for curve progression, according to a literature review ${ }^{7-9)}$. Although the Risser $\operatorname{sign}^{10)}$ has been most widely used as a maturity indicator in clinical practice, controversies remain regarding its accuracy. Classically, Lonstein and Carlson's classification using Risser staging was used to predict curve progression in AIS. In patients with a Risser 0 or 1 , who had an initial Cobb angle between $20^{\circ}$ and $29^{\circ}, 68 \%$ of patients' scoliotic curve deteriorated during growth ${ }^{7}$. However, its predictive accuracy is not always very good. Another major drawback of Risser staging is that there exists considerable debate regarding reverse direction or fragmentation of iliac apophyseal ossification, decreased intra-rater and interrater reliability, and insensitivity of the Risser sign in detecting a rapid growth spurt ${ }^{10,11}$. These findings show that it is of limited use for predicting curve progression. Moreover, there are two slightly different versions of the Risser system in use: one in the United States and another in France. The American version divides the excursion of the apophysis into quarters along the iliac crest, whereas the French version divides the excursion of the apophysis into thirds. The presence of these two different versions leads to confusion when evaluating the adolescent growth process. Additionally, PHV and two-thirds of pubertal growth typically have passed before the first sign of iliac apophysis ossification ${ }^{1)}$. We believe that growth evaluation using only Risser staging is, therefore, not useful.

As we mentioned above, Sanders et al. incorporated hand radiographs and simplified bone age assessments into a more reasonable tool that has been shown to be predictive of PHV. Unlike iliac apophysis growth, the digital epiphyseal region grows continuously until it caps and fuses with the metaphysis. The stage of this capped epiphysis (SSMS 3 ) is tightly coincided with the curve acceleration phase, correlating not with the onset of the growth but with the timing of PHV. However, SSMS staging evaluation requires an assessment of 19 digital epiphyses of the hand besides 


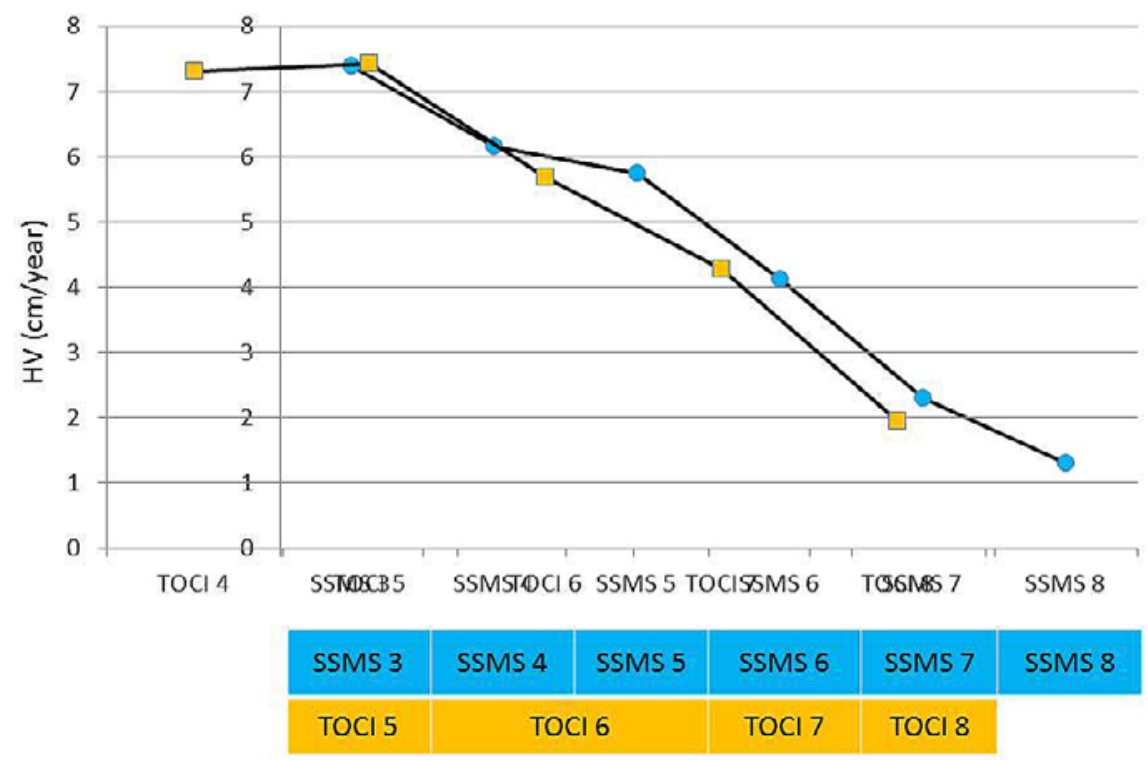

Figure 3. Relationship between HV and SSMS and TOCI stages. When the two line graphs (circles: HV values in each SSMS stage, squares: HV values in each TOCI stage) were superimposed.

$\mathrm{HV}=$ height velocity, SSMS=Simplified Skeletal Maturity Scale, TOCI=Thumb Ossification Composite Index

Table 5. Intraclass and Interclass Correlation Coefficients.

\begin{tabular}{lcc}
\hline \multicolumn{1}{c}{ Intraclass (First versus Second) } & Principal examiner & Another examiner \\
\hline SSMS & $0.991(0.989-0.993)$ & $0.989(0.985-0.991)$ \\
TOCI & $0.992(0.989-0.994)$ & $0.993(0.991-0.995)$ \\
\hline Interclass (Principal versus Another) & 1 st. & 2nd. \\
\hline SSMS & $0.991(0.988-0.993)$ & $0.979(0.973-0.984)$ \\
TOCI & $0.989(0.986-0.991)$ & $0.981(0.976-0.985)$ \\
\hline
\end{tabular}

The values are given as the reliability with $95 \%$ confidence interval in parentheses. SSMS Simplified Skeletal Maturity Scale, TOCI Thumb Ossification Composite Index

the radius, which is complex and cumbersome to use in a busy clinical practice. Recently, we have analyzed the relationship between the magnitude of the Cobb angle at PHV and scoliosis progression: a Cobb angle of $31.5^{\circ}$ when patients were at PHV had the best ROC with $78 \%$ sensitivity, $82 \%$ specificity, and an AUC of 0.93 for predicting curve progression to a magnitude that required surgery ${ }^{12}$. This result is similar to that found in the previous reports ${ }^{1,13)}$. We suggest that a curve progression risk assessment in patients with AIS should include PHV and Cobb angle at PHV along with measures of skeletal and/or non-skeletal maturity.

Since Little et al. first reported that PHV could be used as a simple and reliable clinical marker for the prediction of remaining growth and curve progression of AIS in clinical practice, much attention has been paid to using PHV as a possible predictor of curve progression in patients with AIS. However, PHV itself is only determined retrospectively so its value is not available at the first outpatient clinic visit. To overcome this shortcoming, there have been several studies that were attempting to predict the occurrence of PHV in adolescents. These studies can be categorized based on their method of investigation - anthropometric, equation, or radiograph-based-as a surrogate marker of $\mathrm{PHV}^{14)}$. A single study examined anthropometric-based methods including sitting height, sub-ischial leg length, and foot length ${ }^{15)}$. A few studies mathematically modeled various patient characteristics, namely, anthropometric measures, age, and body mass to predict $\mathrm{PHV}^{16,17)}$. Presently, there are several radiographbased skeletal maturity scoring systems used to gauge the amount of childhood growth and determine the timing of PHV (Table 6). Charles et al. advocated that the olecranon method, which is divided into five stages and is derived from the Sauvegrain method ${ }^{18)}$, has allowed the evaluation of skeletal age from pubertal onset to the timing of PHV. Complete fusion of the olecranon was indicative of $\mathrm{PHV}^{19)}$. Luk et al. proposed the distal radius and ulna classification, which incorporates the whole range of growth phases with 11 radius grades (R1-R11) and nine ulna grades (U1-U9), as an alternative to the maturity parameters discussed ${ }^{20)}$. Medial capping of the distal radius (R7) and early appearance of the 
Table 6. Skeletal Maturity Scoring Systems.

\begin{tabular}{lllrlll}
\hline & \multicolumn{1}{c}{ Authors (Years) } & \multicolumn{1}{c}{ Bone (s) } & Epiphysis & Stage No. & PHV timing $\begin{array}{c}\text { Correlation with } \\
\text { DSA/RUS }\end{array}$ \\
\hline Olecranon method & Charles et al. (2007) & Olecranon & 1 & $5(1-5)$ & Stage 5 & N/A \\
SSMS & Sanders et al. (2007) & Fingers (I-V) \& Radius & $20(19+1)$ & $8(1-8)$ & SSMS 3 & Yes \\
DRU classification & Luk et al. (2014) & Radius \& Ulna & 2 & R1-R11, U1-U9 & R7/U5 & N/A \\
Calcaneal method & Nicholson et al. (2015) & Calcaneous & 1 & $6(0-5)$ & Stage 3/4 & N/A \\
TOCI & Hung et al. (2017) & Thumb & 3 & $8(1-8)$ & TOCI 5 & Yes \\
\hline
\end{tabular}

SSMS Simplified Skeletal Maturity Scale, DRU Distal Radius \& Ulna, TOCI Thumb Ossification Composite Index, N/A not applicable

ulna styloid, with the head of the ulna distinctly defined and denser than the styloid (U5), signified the peak growth spurt. Nicholson et al. demonstrated that calcaneal apophyseal ossification stages (Calcaneal stages 0-5) and SSMS can also be used in combination, offering a better assessment of skeletal maturity with respect to PHV than either system alone $^{21}$. Calcaneal stage 3 and SSMS 2 both occur 0.9 years before PHV, whereas calcaneal stage 4 and SSMS 3 both occur approximately 0.5 years after PHV. Recently, a validation study showed that the new simplified TOCI system (based on the epiphyses of three bones: the distal and proximal phalanges together with the adductor sesamoid bone) demonstrated simplicity, excellent reliability, and high accuracy for predicting skeletal maturity, comparable with the SSMS system ${ }^{22}$. Their study also showed that the mean PHV was $7.4 \mathrm{~cm} /$ year and occurred at a mean age of 11.9 years, which is consistent with the previous study of Chazono et al. ${ }^{23}$. In the present study, we investigated the relationship between the SSMS and TOCI stages in Japanese female patients with AIS. We found that their TOCI stage rating increased in parallel with increases in their SSMS stage rating, indicating a significant correlation between the two scoring systems. Regarding the relationship between HV values and each SSMS and TOCI stage, the mean value of HV decreased as SSMS and TOCI stages increased. Not only were both the SSMS 3 and TOCI 5 stages possibly associated with the timing of PHV, but the relationship between each SSMS and TOCI stage with the value of HV was also elucidated. In the present study, SSMS 3, SSMS 4, SSMS 5, SSMS 6, SSMS 7, and SSMS 8 corresponded with $7.4,6.2,5.8,4.1,2.3$, and $1.3 \mathrm{~cm} /$ year of $\mathrm{HV}$ values and TOCI 4 , TOCI 5, TOCI 6 , TOCI 7 , and TOCI 8 with 7.3 , $7.4,5.7,4.3$, and $1.7 \mathrm{~cm} /$ year of $\mathrm{HV}$ values. Interestingly, the relationship between the SSMS and TOCI stages in the present study was consistent with the findings of Hung's study: SSMS 3 corresponds with TOCI 5; SSMS 4 and SSMS 5 with TOCI 6; SSMS 6 with TOCI 7; and finally, SSMS 7 with TOCI $8^{22)}$. Knowing that HV values from the degree of skeletal maturity were obtained from hand radiographs, we can speculate on the adolescent growth period for AIS patients. The following analogy may help illuminate our findings: when the degree of patients' skeletal maturity is at SSMS 3 or TOCI 5, it seems that they are at the top of a mountain, with a steep decline in ahead of them, whereas when they are at SSMS 7 or higher or TOCI 8 , they are at the foot of the mountain, finishing mountain-climbing during the adolescent growth period.

To the best of our knowledge, this study is the first to determine the quantification of $\mathrm{HV}$ in each SSMS and TOCI staging. However, one of the possible limitations of this study is the relatively small number of patients. Nonetheless, a total of 6118 epiphyses obtained from 266 hand radiographs were meticulously scored for 95 consecutive patients in this study. Thus, we believe that we can guarantee the reliability of these classifications to some extent. Another limitation is that only a single examiner has judged the skeletal maturity staging according to SSMS and TOCI systems. A further investigation from multicenter institutions with a bigger sample size would be required to validate the reliability and usefulness of these scales in clinical setting.

\section{Conclusion}

The newly proposed TOCI staging system, which involves the assessment of two thumb epiphyses and the sesamoid bone instead of the 19 digital epiphyses plus radius used in the SSMS system, has been shown to be a simple, reliable, and accurate method to predict skeletal maturity in patients with AIS. The TOCI may serve as a simpler marker of skeletal maturity and an alternative to the SSMS staging system. For now, it is indispensable for clinicians to predict the value of $\mathrm{HV}$ and determine the "milestone" for the growth period in patients with AIS using hand radiographs at regular outpatient clinic visits.

Conflicts of Interest: The authors declare that there are no relevant conflicts of interest.

Ethical Approval: Certified No.2-2 by institutional review board (IRB) of Utsunomiya National Hospital

Informed Consent: Informed consent was obtained by all participants in the present study.

\section{References}

1. Little DG, Song KM, Katz D, et al. Relationship of peak height velocity to other maturity indicators in idiopathic scoliosis in girls. J Bone Joint Surg Am. 2000;82(5):685-93.

2. Sanders JO, Browne RH, Cooney TE, et al. Correlates of peak height velocity in girls with idiopathic scoliosis. Spine (Phila Pa 
1976). 2006;31(20):2289-95.

3. Sanders JO, Browne RH, McConnell SJ, et al. Maturity assessment and curve progression in girls with idiopathic scoliosis. J Bone Joint Surg Am. 2007;89(1):64-73.

4. Sanders JO. Maturity indicators in spinal deformity. J Bone Joint Surg Am. 2007;89(Suppl 1):14-20.

5. Hung ALH, Chau WW, Shi B, et al. Thumb ossification composite index (TOCI) for predicting peripubertal skeletal maturity and peak height velocity in idiopathic scoliosis. J Bone Joint Surg Am. 2017;99(17):1438-46.

6. Sanders JO, Khoury JG, Kishan S, et al. Predicting scoliosis progression from skeletal maturity: a simplified classification during adolescence. J Bone Joint Surg Am. 2008;90(3):540-53.

7. Lonstein JE, Carlson JM. The prediction of curve progression in untreated idiopathic scoliosis during growth. J Bone Joint Surg Am. 1984;66(7):1061-71.

8. Soucacos PN, Zacharis K, Gelalis J, et al. Assessment of curve progression in idiopathic scoliosis. Eur Spine J. 1998;7(4):270-7.

9. Tan KJ, Moe MM, Vaithinathan R, et al. Curve progression in idiopathic scoliosis: follow-up study to skeletal maturity. Spine (Phila Pa 1976). 2009;34(7):697-700.

10. Risser JC. The iliac apophysis: an invaluable sign in the management of scoliosis. Clin Orthop. 1958;11:111-9.

11. Shuren N, Kasser JR, Emans JB, et al. Reevaluation of the use of the Risser sign in idiopathic scoliosis. Spine (Phila $\mathrm{Pa}$ 1976). 1992;17(3):359-61.

12. Chazono M, Tanaka T, Marumo K, et al. Significance of peak height velocity as a predictive factor for curve progression in patients with idiopathic scoliosis. Scoliosis. 2015;10(Suppl 2):S5.

13. Sitoula P, Verma K, Holmes Jr, et al. Prediction of curve progression in idiopathic scoliosis. Spine (Phila Pa 1976). 2015;40(13): 1006-13.

14. Mills K, Baker D, Pacey V, et al. What is the most accurate and reliable methodological approach for predicting peak height velocity in adolescents? A systematic review. J Sci Med Sport. 2017;20
(6):572-7.

15. Busscher I, Gerver WJM, Kingma I, et al. The growth of different body length dimensions is not predictive for the peak growth velocity of sitting height in the individual child. Eur Spine J. 2011; 20(5):791-7.

16. Busscher I, Kingma I, de Bruin R, et al. Predicting the peak growth velocity in the individual child: validation of a new growth model. Eur Spine J. 2012;21(1):71-6.

17. Malina RM, Claessens AL, van Aken K, et al. Maturity offset in gymnasts: application of a prediction equation. Med Sci Sports Exerc. 2006;38(7):1342-7.

18. Sauvegrain J, Nahum H, Bronstein H. Study of bone maturation of the elbow. Ann Radiol (Paris). 1962;5:542-50. French.

19. Charles YP, Dimeglio A, Canavese F, et al. Skeletal age assessment from the olecranon for idiopathic scoliosis at Risser grade 0 . J Bone Joint Surg Am. 2007;89(12):2737-44.

20. Luk KD, Saw LB, Grozman S, et al. Assessment of skeletal maturity in scoliosis patients to determine clinical management: a new classification scheme using distal radius and ulna radiographs. Spine J. 2014;14(2):315-25.

21. Nicholson AD, Sanders JO, Liu RW, et al. The relationship of calcaneal apophyseal ossification and Sanders hand scores to the timing of peak height velocity in adolescents. Bone Joint J. 2015;97$\mathrm{B}(12): 1710-7$.

22. Hung ALH, Shi B, Chow SKH, et al. Validation study of the thumb ossification composite index in idiopathic scoliosis. J Bone Joint Surg Am. 2018;100(13):e88(1-9).

23. Chazono M, Soshi S, Kida Y, et al. Height velocity curves in female patients with idiopathic scoliosis. Studies in Health Technology and Informatics. 2012;176:202-5.

Spine Surgery and Related Research is an Open Access journal distributed under the Creative Commons Attribution-NonCommercial-NoDerivatives 4.0 International License. To view the details of this license, please visit (https://creativeco mmons.org/licenses/by-nc-nd/4.0/) 